\title{
Mobility assessment in older people: new possibilities and challenges
}

\author{
Wiebren Zijlstra $\cdot$ Kamiar Aminian
}

Published online: 6 February 2007

(C) Springer-Verlag 2007

\begin{abstract}
A major challenge for researchers and clinicians who address health issues in the ageing population is to monitor functioning, and to timely initiate interventions that aim to prevent loss of functional abilities and to improve the quality of life of older people. With the progress of technologies in the last decades, methods have become available that use body fixed sensors (BFS) to measure aspects of human performance under real-life conditions. These methods are based on the use of miniaturised and integrated sensors in combination with lightweight, small measuring devices that both can be carried on the body without interfering with normal behaviour. This paper addresses the potential relevance of new technology for monitoring motor function in older people, thereby specifically focusing on mobility assessment. After a short introduction with background information about BFS based technology, this paper identifies areas of particular relevance, and discusses the application of ambulatory techniques for long-term monitoring of daily physical activity, fall detectors, fall risk evaluation, and assessment of motor performance such as gait and balance control. Examples are given how these
\end{abstract}

W. Zijlstra $(\bowtie)$

Center for Human Movement Sciences,

University Medical Center Groningen,

University of Groningen, P.O. Box 196,

9700 AD Groningen, The Netherlands

e-mail:w.zijlstra@med.umcg.nl

\section{K. Aminian}

Ecole Polytechnique Fédérale de Lausanne,

Laboratory of Movement Analysis and Measurement,

Lausanne, Switzerland techniques can become clinically relevant, particularly in the context of fall interventions for older people.

Keywords Mobility · Assessment ·

Activity monitoring $\cdot$ Ageing $\cdot$ Body fixed sensors

\section{Introduction}

In the ageing population, safe and independent mobility is often hindered by age-related pathology, such as osteo-arthrosis, stroke, Parkinson's or Alzheimer's disease. Even in the absence of overt pathology, motor functioning [cf. International Classification of Functioning (ICF) by the World Health Organisation, Geneva (see http://www.who.int/classification/icf)] can deteriorate, as is illustrated by the incidence and impact of falls in the European ageing population (Skelton and Todd 2004). Approximately $30 \%$ of persons older than 65 years fall each year, and after the age of 75 fall rates are even higher (O'Loughlin et al. 1993). Between 20 and $30 \%$ of those who fall suffer injuries that reduce mobility and independence and increase the risk of premature death. Falls are associated with significant social and psychological consequences, as people lose confidence and become isolated and restrict their activity. The reduction of physical activity caused by pathology, and/or psychological factors such as fear of falling, has serious physiological consequences. Activity avoidance may lead to reductions in muscle strength, joint mobility, and cardio-respiratory function. In addition, the lack of experience in performing certain activities leads to a deconditioning, which increases the likeliness of unsafe performance of activities. Thus, a downward spiral 
commences which can only be prevented by timely interventions that improve motor functioning and facilitate safe performance of activities (Fig. 1).

To further the development of effective fall prevention programmes, the European Commission has funded the Prevention of Falls Network Europe (ProFaNE) (see Skelton et al. 2004). One of the issues that ProFaNE is concerned with is the clinical assessments that are required for those "at risk" of falls. For researchers and clinicians addressing health issues in the ageing population, it still is a major challenge to effectively monitor functioning, and prevent loss of functional abilities in order to maintain or improve quality of life. The development of effective interventions requires an understanding of the mechanisms that underlie the loss of functioning, but also instruments and procedures for monitoring functioning before, during and after an intervention. This paper addresses the potential contribution of novel body-fixed-sensor based technology to monitoring functioning in older people. The paper specifically focuses on different aspects of mobility assessment. After a short introduction with background information about body-fixedsensor based technology, this paper identifies areas of particular relevance, and discusses the application of ambulatory techniques for long-term monitoring of daily physical activity, fall detectors, fall risk evaluation, and assessment of motor performance such as gait and balance control. Examples are given how these techniques can become clinically relevant, particularly in the context of fall interventions for older people.

\section{Body-fixed-sensor based technology}

With the progress of technologies in the last decades, methods have become available that use body-fixedsensors (BFS) to measure aspects of human performance under real-life conditions (Aminian and Najafi 2004). These methods are based on the use of miniaturised and integrated sensors in combination with light-

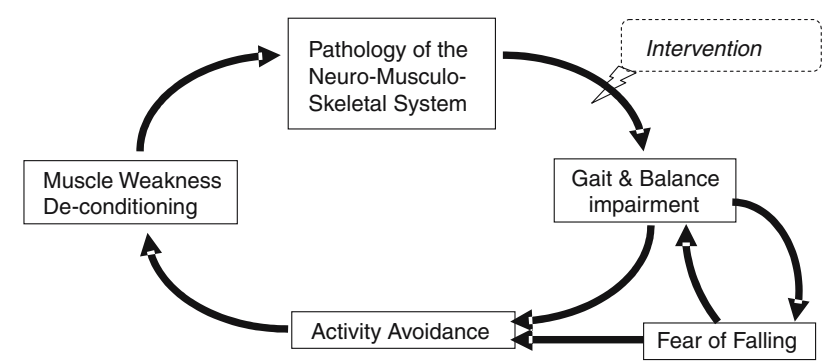

Fig. 1 Schematised representation of factors contributing to increases or decreases in fall risk weight, small measuring devices that can be carried on the body without interfering with normal behaviour.

Main types of body fixed sensors

Accelerometers can be used to measure accelerations of body segments, and the effect of gravity on the segment. An accelerometer consists of a small mass inside an accelerometer case that is connected to the case by a beam. The system can be regarded as a dampened spring; any acceleration of the sensor results in a deformation of the beam that connects mass and case. The deformation is transduced to a change of electrical impedance (resistive or capacitive accelerometer) or charge generation (piezoelectric accelerometer). In the absence of movement, the former type of accelerometer also measures the effect of gravitation on the internal mass. Thus, the inclination of the sensor with respect to the vertical axis can be calculated.

By attaching accelerometers on one or more body segments (e.g., trunk, thigh, and shank), the body posture at rest (i.e., standing, sitting, and lying) can be recognized. For example, when the longitudinal axis of a 3D accelerometer on the trunk measures approximately $1 \mathrm{~g}$, its orientation is upward, and the subject must be standing or sitting. When it measures $0 \mathrm{~g}$, the trunk is horizontal and the subject must be lying (see Fig. 2). In the latter situation, the combination of acceleration signals (i.e., vertical, forward and left/ right accelerations) give information about whether the subject is lying prone or supine, or on the left or right side. During activities, movements of body segments induce inertial acceleration and a variable gravitational component depending on the change of segment inclination with respect to the vertical axis. Both acceleration components are superimposed and their separation is necessary for a proper analysis of the movement.

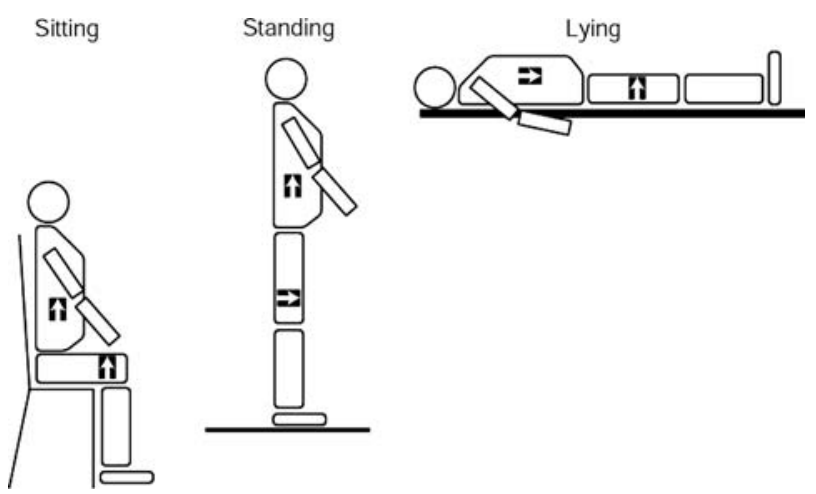

Fig. 2 During rest the orientation of body segments can be detected by accelerometers 
Miniature gyroscopes use a vibrating mechanical element to sense angular velocity. All vibratory gyroscopes are based on the transfer of energy between two vibration modes of a structure caused by the Coriolis force. The Coriolis effect is an apparent force that arises in a rotating reference frame and is proportional to the angular rate of rotation. The vibration force can be induced using piezoelectric, electromagnetic and electrostatic energy. The Coriolis force can be detected using piezoelectric, resistive or capacitive effect. Gyroscopes have been developed particularly for the automotive and navigation market as well as in consumer products such as anti-jitters compensation for video cameras. Although the use of gyroscope for human motion capture is still rather new and under development, gyroscopes can be considered a promising tool in this field. Human motion consists mainly of segment rotations around joints. Unlike accelerometers, there is no influence of gravitation on the signal measured by gyroscopes. The gyroscope can be attached to any part of a body segment: as long as its axis is parallel to the measured axis, the angular rotation is still the same along this segment. Rotation angles can be estimated from angular velocity by simple numeric integration. However, due to integration drift, it is problematic to estimate absolute angles from angular velocity data.

Earth magnetic field sensors or magnetometers can be used to estimate changes in the orientation of a body segment relative to the magnetic North. These sensors use the magneto-resistive effect, which corresponds to a change of resistance under a (change in) magnetic induction. Earth magnetic field sensors can be used to determine segment orientation around the vertical axis. Hence, they provide information that cannot be determined from accelerometers or by the integration of gyroscope signals. A disadvantage of magnetometers is their sensitivity to nearby ferromagnetic materials (e.g., iron) and magnetic fields other than that of the earth magnetic field (e.g., electro-magnetic fields produced by a TV screen).

Pressure sensors or foot switches attached to the shoe or foot sole can be used to detect contact of the foot with the ground. By detecting heel and/or toe contacts, the sensors can identify different movement phases during walking [i.e., swing, stance and bipedal stance phases (see Fig. 3)], or they can provide pressure distribution of the foot during stance phases. These techniques allow the measurement of longer periods of walking with many subsequent stride cycles.

Goniometers can be used to directly measure changes in joint angle. Different kinds of goniometers have been reported, but, regardless of sensor type, the basic principle is the same: two axes of a goniometer are attached to the proximal and distal segments of a joint and a voltage is generated that is proportional to the angle between these two axes. Sensor attachment, accuracy, and the lack of robustness of the sensors are major drawbacks of using goniometers.

\section{Signal recording, processing and modelling}

Main features to be considered for the design of recording systems that are carried on the body for long-term recording are: size, weight, robustness, power consumption, memory size, the resolution of the analog-to-digital (A/D) conversion process, number of channels, bandwidth and sampling rate. Progress has been made in near to all relevant aspects of the design of such ambulatory systems. Particularly, the availability of powerful micro-controllers, miniature sensors, high capacity memory and small batteries allow ambulatory monitoring systems that are relatively simple to carry and do not, or only minimally, disturb the subject in his or her daily activities (see Fig. 4).

Before data measured by BFS can be interpreted, further calculations and analyses are necessary. First, the kinematics of the measured body segments must be calculated, and secondly further analyses must yield (clinically) relevant information from the movement data. Moreover, measured data might even be distorted by limited accuracy, electronical noise, drift and/or movement artefacts. Therefore, advanced signal processing is necessary to extract relevant information from BFS data. An important area of further development of BFS based technology for monitoring human movements lies in the smart use of measured signals. Several examples may illustrate this point.
Fig. 3 Foot pressure sensors or foot switches attached under the foot allow estimation of onset and termination of stance and swing phases during walking

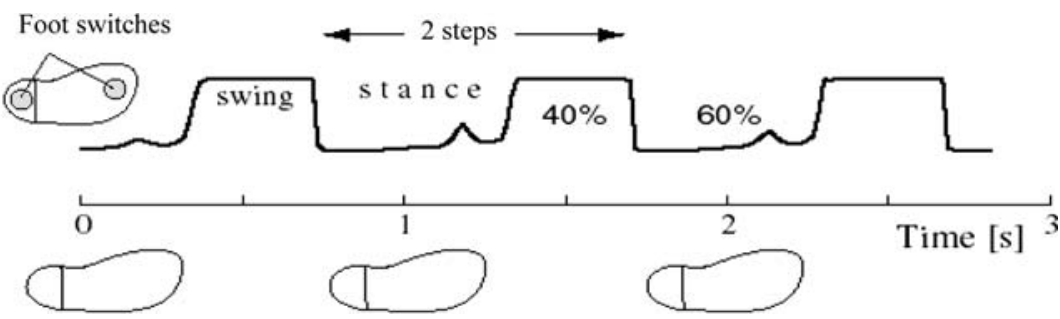




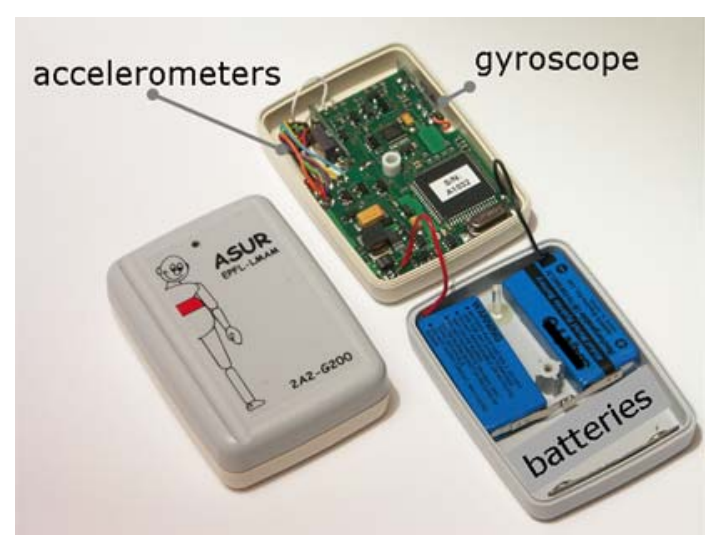

Fig. 4 Example of an ambulatory recording device, including sensors, electronics, memory and batteries

First, signal processing is needed to overcome specific limitations of BFS. Sensors are never perfect, they come with their specific advantages and disadvantages. Therefore, appropriate signal processing methods are required in order to estimate body kinematics with an acceptable accuracy. In addition, before further analysis data must often be transformed to an inertial reference frame. Accelerometers and gyroscopes measure within a local frame of reference. The orientation of this local reference frame depends on the orientation of the body segment to which the sensor is attached. For further analyses and interpretation of the data, it is often desirable to determine body movement in an absolute (or inertial) frame of reference. To transform the BFS data from a local segment oriented reference frame to an inertial reference frame requires data from different BFS types and adequate signal processing. An important recent development is the fusing of data from different sensor types. Using new signal processing algorithms (Luinge and Veltink 2005), and hybrid sensors, consisting of accelerometers, gyroscopes (Favre 2006) and accelerometers, gyroscopes magnetometers (Roetenberg et al. 2005), some disadvantages of individual sensor types can be overcome. The fusing approach allows a BFS based analysis of joint movements and segment movements in an inertial frame of reference.

Secondly, advanced signal processing techniques are necessary for analysing time varying properties of kinematic data. Only when a certain body posture is maintained do BFS measure stationary signals, during most activities signals are non-stationary. Examples of nonstationary signals include the acceleration pattern during postural transitions such as sit-to-stand or stand-tosit, and walking with varied velocity. In these situations, the information of interest is often a combination of features that are well localized in time and frequency domains. In contrast to Fourier transform, ${ }^{1}$ which is not suited to characterize the temporal distribution of singularities and transient events, the wavelet transform $^{2}$ is an optimal technique for describing singularities in signals. In addition, instead of decomposing the kinematic signal to sinusoids, wavelet transform uses a suitable basic function, which is more similar to the typical patterns in kinematic data. Several investigators have shown the advantages of wavelet transform for the analysis of kinematic signals (Sekine et al. 2000; Lakany 2000; Paraschiv-Ionescu et al. 2004; Najafi et al. 2002).

It should be noted that the choice for specific signal processing very much depends on the specific goal of the measurements, and not all analyses of human activities need a complete description of movements of all body segments. The same activity, for example gait, may be analysed differently depending on whether overall measures (e.g., duration of walking and estimated walking distance) or specific measures (e.g., joint rotation) are required. Depending on knowledge of the specific movements under study, signal analyses can be simplified by focussing on some relevant aspects of the measured signal or by using knowledge of the specific constraints of the movements.

\section{Potential relevance of using BFS for analysing human movements}

Despite the fact that they are often taken as the goldstandard, laboratory-based measures of human movement do have some serious limitations. Typical laboratory equipment consists of a combination of force plates, treadmill or instrumented walkway, and optical, sonic or magnetic motion capture systems. These instruments provide objective and quantitative measures of movement, but using them requires a permanent laboratory and a specialized and technically educated staff, which carries out the time consuming measurements and data analyses. A major disadvantage of laboratory studies is that human movements are studied in an environment that does not present the subject with the wealth of information or stimuli of a natural environment. In a laboratory, the subject

\footnotetext{
${ }^{1}$ Fourier transform; a signal analysis technique that is based on the fact that any periodic signal can be described as the sum of a limited number of sinusoidal functions with different frequencies and amplitudes.

${ }^{2}$ Wavelet analysis; a signal analysis technique that is based on the description of a "typical" signal shape (wavelet) with a limited number of coefficients.
} 
moves inside an artificial environment and motion can only be captured within the limited work space of motion capture systems (e.g., a camera system). Unless a treadmill is used, only a few subsequent steps can be measured during walking. Thus, it can be questioned whether such data reflects the way habitual activities are carried out in daily life, and whether typical laboratory experiments can provide clinically relevant measures of motor functioning.

From the above, it is clear that the feasibility of using laboratory-based measures of human movement for routine-use in a regular health care setting is very limited. At present, it is common practice to use interviews (e.g., the 7 day recall (Sallis et al. 1985), questionnaires (e.g., SF-36 (Ware and Sherbourne 1992); the Physical Activity Scale for the Elderly [PASE, Washburn et al. 1993); the Short Questionnaire to Assess Health-enhancing physical activity (SQUASH, Wendel-Vos et al. 2003)] or observational instruments [e.g., Rivermead Mobility Index (Collen et al. 1991)] to acquire data about physical health in relation to quality of life, physical activity, and mobility. Motor performance can also be assessed by simple field tests such as the Berg's Balance Scale (Berg et al. 1992), or the Timed Up and Go test (Podsiadlo and Richardson 1991). Although questionnaires, observational methods, and simple field tests may yield clinically relevant information and often have the advantage of ease-of-use, the sensitivity of these methods for quantifying changes in movement or activity patterns is limited. The latter is particularly problematic when the activity patterns of sedentary older people are to be assessed, since these activities often consist of sitting, lying, standing and short indoor walks. Thus, the activities are characterised by (very) low intensity and small changes in activity levels cannot be captured.

BFS based measures have important advantages over both laboratory based measures and the use of questionnaires or observational instruments. BFS provide objective data of actual movement behaviour. While minimally interfering with habitual activities, BFS based data can be obtained over long time periods and in a natural environment. In addition, BFS also provide data that cannot be obtained within the laboratory or by questionnaires (for example, the walking distance during the day, or an assessment of gait variability which requires data collection over a large number of subsequent stride cycles). Moreover, BFS based methods can be developed in such a way that they meet the requirements for application on a routine base in regular health care, for example by nurses, occupational therapists or physical therapists. Thus, the use of BFS may ultimately provide the clinician or scientist with "the" tool for obtaining objective measures of motor functioning in daily life.

\section{Applications of BFS based technology for mobility assessment}

Although at present, the full potential of BFS based technology has not yet been realised, a number of relevant applications is now reaching maturity. This section will highlight some applications that are particularly relevant with respect to mobility assessment in the ageing population.

Estimating activity level and activity related energy expenditure

Accelerometers have long been used to estimate activity level and activity related energy expenditure. The underlying assumption is that activities require accelerations (and decelerations) of body segments, and the production of these accelerations is to a large extent responsible for activity related energy expenditure. In a simple way, activity level and energy expenditure can be estimated by counting the number of steps during a certain time period. Step counters are available in different types, and have the advantage of being small, easy-to-use and affordable. However, step counters only quantify activities that include the typical pattern of accelerations associated with stepping (or jumping). A more refined approach is to use measures of the acceleration of body segments in one or more directions to estimate energy expenditure. For example, studies of trunk accelerations and energy expenditure during different activities have shown accurate estimations of energy expenditure during level walking in young adults based on the rectified and integrated acceleration signal (Bouten et al. 1994). However, large errors occurred when a calibration based on level walking was used to estimate energy expenditure during inclined walking (Terrier et al. 2001), and estimations are less accurate for other activities than walking. For other activities, accelerometry based estimations of energy expenditure can be improved by placing accelerometers on several different body segments (e.g., Schwartz et al. 2000). Since BFS can be used to assess the frequency and duration of body postures and activities (see next section), it is also possible to estimate activity related energy expenditure based on the occurrence of specific postures and activities. 
Activity monitoring

Since the early nineties a number of papers have demonstrated the possibility of categorizing different postures and activities from BFS signals (e.g., Bussmann et al. 1995; Veltink et al. 1996; Aminian et al. 1999). These first activity monitors were primarily based on the use of multiple accelerometers on trunk and leg segments. Body postures such as sitting, standing, or lying were detected based on the static component of the accelerometer signals (cf. preceding section), and activities like walking, cycling, or stair walking were detected based on activity specific information in both static and dynamic components of the acceleration signals. Recently, only one sensor on the chest was used to detect different activities and transitions from one activity to the other. The sensor consisted of two accelerometers with one gyroscope. Sitting and standing positions were identified by recognizing the different nature of sit-to-stand and stand-to-sit transitions (Najafi et al. 2003a, b). The detection of postural transitions allows the classification of body postures before and after the transition, and also an analysis of the transition itself.

Generally, video- and observation-based validations of BFS based methods for detecting body postures and activities have shown reasonable to good results in terms of sensitivity and specificity (for example see Paraschiv-Ionescu et al. 2004), but correct identification of some activities (i.e., stair ascend and descend) has proven to be more difficult than others. In general, the more sensors are used, the higher the probability of a correct classification. However, it is clear that for practical reasons, it may be desirable to have as few sensors as possible.

After measuring BFS data over a long duration (e.g., $12 \mathrm{~h}$ periods over a number of subsequent days), a post hoc analysis reveals frequency and duration of specific postures and activities (Fig. 5). Thus, individual activity patterns can be objectively quantified instead of subjectively based on questionnaires or diary reports. It can be expected that the exclusion of subjective noise in activity reports (e.g., recall problems and the tendency of respondents to give "socially desired" answers) and the increased accuracy and resolution of a BFS based assessment of activity patterns will pave the way for gaining insight into the relationships between activity patterns, quality of motor performance and measures that indicate self-perception of functioning (e.g., fear of falling).

At present, the feasibility and relevance of using activity monitors have been demonstrated in numerous studies. For example, intervention studies that address fall risk in older people (e.g., Najafi et al. 2002), studies of the effects of interventions in Parkinson's disease patients (Keijsers et al. 2006), pain (Buchser et al. 2005) or patients after orthopaedic surgery (Morlock et al. 2001). Although these and other studies demonstrate the relevance of activity monitors, their full potential for evaluating changes in activity patterns as a result of interventions has not yet been attained.

Within the field of fall prevention, activity monitoring and BFS based assessments of motor performance may help in unravelling the complex interrelationships between fall risk, fear of falling, activity avoidance, and motor (dis)abilities. Depending on individual risk factors, fear of falling and activity avoidance may be realistic or unrealistic, and to be effective fall prevention strategies need to be tailored to individual risk profiles. A paradoxical result of some fall prevention studies has been that the number of falls increased during and after the intervention. One explanation for such findings could be that as a result of the intervention participants become more active and hence increase their risk
Fig. 5 Typical results for long-term monitoring of physical activity showing frequency and duration of specific postures and walking periods

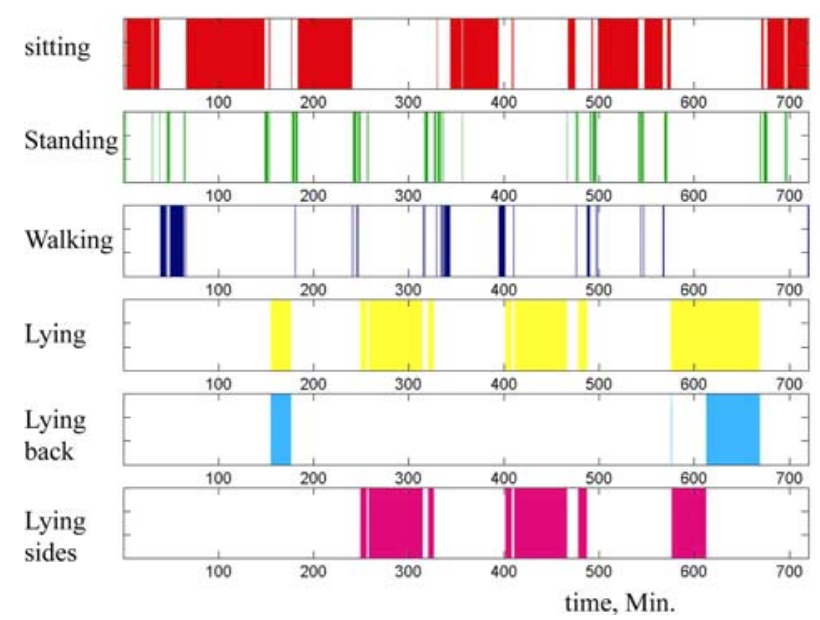


of falling. This effect would be similar to a car driver increasing the likeliness of an accident by increasing his/her mileage. Since the possibility to increase activity levels can be considered as a contribution to quality of life, it may be more appropriate to express fall risk related to activity level and not in terms of number of falls per unit of time. Here the use of BFS could support the development of effective fall prevention strategies that are based on insight in the individual interrelationships between activity patterns and number of falls.

\section{Fall detectors}

Reliable methods for detecting falls are extremely relevant in two aspects; first, in order to signal the occurrence of a fall so that suitable measures can be initiated when assistance is needed (i.e., fall alarms), and secondly, to obtain a valid documentation of falls (fall reports). At present, there is no unanimously accepted method for reporting falls. Fall definitions vary (Hauer et al. 2006), and the usual method of verbal reporting by fallers, spouses, or nurses, includes several error sources (e.g., Kanten et al. 1993). Automatic fall detection is important in overcoming the shortcomings of non-automatic home alarm systems that depend on the subject being alert enough to use the alarm system after a serious fall. However, for an automatic home alarm system, it is necessary that falls are detected with enough sensitivity, and without generating false alarms (i.e., high specificity). BFS based fall detectors may be the basis for reliable fall reports and automatic fall alarm systems (e.g., see Lindemann et al. 2005), but at present, there is very little published information about BFS based fall detectors.

\section{Assessment of balance and gait}

An increasing number of papers demonstrate the use of BFS based methods for analysing gait and other balance tasks. Important spatio-temporal gait parameters such as walking speed, step length and frequency, duration of swing and stance phases can be determined from BFS on leg or trunk segments (e.g., Hausdorff 1995; Aminian et al. 2002; Zijlstra 2004). These basic gait parameters can be complemented with additional spatio-temporal parameters that indicate the joint kinematics, e.g., flexion-extension patterns in ankle, knee or hip (Dejnabadi et al. 2005, 2006) or joint dynamics (Zijlstra and Bisseling 2004). In addition, BFS can be used to quantify the performance of balance tasks such as the transitions between postures and activities. The following paragraphs demonstrate that obtaining measures of motor functioning by BFS can provide a significant contribution to estimating fall risk and the evaluation of intervention effects.

Most falls occur during activities that require a change of body position, e.g., standing up, sitting down, initiating walking, or walking (e.g., Bergland et al. 1998). Performance of the sit-to-stance transfer requires the ability to maintain balance while producing enough muscle force to raise the body's centre of mass from a seated to a standing position (e.g., Schenkman et al. 1996; Lord et al. 2002). Studies of rising from a chair have demonstrated age effects on the sit-tostance performance (e.g., Pai and Rogers 1990), and an association of task performance with muscle force in the leg extensors (e.g., Ploutz-Snyder et al. 2002). By using BFS on the thorax for analysing body transfers, it has been demonstrated that the duration as well as the variability of rising from a chair and sitting down can distinguish older people with a low or high fall risk (Najafi et al. 2002).

Balance during different standing conditions and during gait have been assessed using different BFS configurations. The use of gyroscopes for assessing trunk rotations can identify a balance disorder and possibly distinguish between different types of balance disorder (Allum et al. 2001, 2005). Large leftright rotations (i.e., "roll") of the trunk as measured by a gyroscope during walking has been used to distinguish older fallers from non-fallers (de Hoon et al. 2003). A BFS based gait analysis, showed that spatiotemporal gait parameters, such as walking speed, step length and the duration of bipedal stance, correspond well to the scores of older people with high and low fall-risks, based on the Tinetti score. However, the Tinetti score is much less discriminatory than the gait parameters for subjects with a moderate risk of falling (Najafi et al. 2003a, b). Estimations of gait variability by means of an ambulatory system with a tri-axial accelerometer on the lower trunk, showed lower left-right, but higher vertical and forward trunk variability in frail elderly when compared to a fit control group (Moe-Nilssen and Helbostad 2005). The trunk variability measures correctly classified $80 \%$ of the subjects into their respective group ( sensitivity $=0.75$, specificity $=0.85)$. By using pressure sensors in the shoe (Hausdorff et al. 1995), it could be demonstrated that gait inter-cycle variability is significantly higher in faller than non-fallers and young subjects (Hausdorff et al. 1997). Moreover, using the same sensors, it could be demonstrated that the long-range variability in gait patterns is strongly related to degree of functional impairment (Hausdorff et al. 2001). 
Balance control during standing and walking is to a high degree an automatic process, controlled by subcortical brain regions. However, under difficult circumstances, or when the automatic control process is less optimal, e.g., because of pathology, inactivity or covert reasons, cognitive resources are required for an optimal task performance (Mulder et al. 2002). Falls often seem to occur during activities when attention needs to be divided between tasks, as illustrated by the wellknown phenomenon "stops walking when talking" (Lundin-Olsson et al. 1997). Use of the so-called dual task assessment paradigm, which requires the performance of a primary (motor) task together with a secondary (cognitive) task, has now demonstrated that balance control is demanding more attention in older people than in young adults (e.g., Woolacott and Shumway-Cooke 2002) An inability to divide attention between postural control and a second task might be a risk factor for falls, and some studies indicate that this is indeed the case. For example, a gait analysis using gyroscopes and a "dual task" paradigm (backward counting during walking) was able to classify older and young subjects (Beauchet et al. 2003). Dual task performance increased stride-to-stride variability in older subjects (Dubost et al. 2006). This strongly suggests the involvement of higher cortical regions for the motor control of gait in this population.

\section{Future perspective and remaining challenges}

At present, the use of BFS for mobility assessment particularly serves the (clinical) researcher with an interest in motor functioning. As is customary for work in a research setting, many of the studies that use BFS are based on custom-made sensor configurations and signal analyses. The commercial availability of BFS based measurement systems is rapidly improving, but still somewhat limited, and there are still some remaining questions with regard to optimal solutions for sensor configuration and signal processing for specific applications such as fall detection, activity monitoring and balance assessments.

Although our examples in the previous sections demonstrate that clinically relevant applications of BFS based technology are available, routine-use in health care requires that some specific requirements are fulfilled. The main motivation for introducing a new clinical practice will always be the existence of evidence that the new approach will significantly contribute to clinical practice, for example by improving diagnosis, prognosis or treatment, or by a cost reduction in comparison to existing clinical practice.
Although evidence exists for the clinical relevance of specific BFS based mobility assessments, further clinical research is needed for the development of evidence-based applications of the new technology. Therefore, the most important remaining challenge in realising the full potential of BFS is the development of evidence based clinical applications that are commercially available. This development requires a close interaction of clinicians, engineers, and industry.

One area where BFS are becoming relevant is related to the developments of assistive technology in the home environment, i.e., domotica and ambient assisted living, and the use of so-called smart clothes that carry sensors that monitor bodily functions (Axisa et al. 2005; Marculescu et al. 2003). Technological advances allow the use of BFS based technology in combination with advanced ICT solutions to effectively monitor and assist older people in their home environment. Based on such technology, interventions can be introduced in the home environment, and tailored to the individual needs. This new approach allows "telecare" solutions where, from a distance, medical professionals monitor older people in their home environment and assist with tailored interventions. Although the technology to realise tele-care solutions is available, the approach still needs to be put in practice by developing applications that are convincing and practicable.

\section{In conclusion}

The preceding sections demonstrate current possibilities for mobility assessment based on BFS. The specific examples illustrate the clinical relevance of the new methods for the field of fall prevention. However, these recent developments have not yet come to an end. Further technical developments, e.g., miniaturisation, clever sensor configurations, and improvements with regard to signal processing, can be expected. Before the new technology will be implemented on a large scale, for example in the home environment or for routine-use in health care settings, evidence-based applications need to be developed and made available on the market.

Acknowledgment The authors are participants in the ProFaNE thematic network, which is a project in Key Action \#6 (The Ageing Population and their Disabilities), part of the European Commission's Fifth Framework, Quality of Life and Management of Living Resources Programme, funded by the European Commission (QLRT-2001-02705). The content of the manuscript does not represent the opinion of the European Community, and the Community is not responsible for any use that might be made of the information presented in the text. 


\section{References}

Allum JH, Adkin AL, Carpenter MG, Held-Ziolkowska M, Honegger F, Pierchala K (2001) Trunk sway measures of postural stability during clinical balance tests: effects of a unilateral vestibular deficit. Gait Posture 14:227-237

Allum JHJ, Carpenter MG (2005) A speedy solution for balance and gait analysis: angular velocity measured at the centre of body mass. Curr Opin Neurol 18:15-21

Aminian K, Robert Ph, Buchser E, Rutschmann B, Hayoz D, Depairon M (1999) Physical activity monitoring based on accelerometry: validation and comparison with video observation. Med Biol Eng Comput 37:304-308

Aminian K, Najafi B, Bula C, Leyvraz PF, Robert P (2002) Spatio-temporal parameters of gait measured by an ambulatory system using miniature gyroscopes. J Biomech 35(5):689-699

Aminian K, Najafi B (2004) Capturing human motion using bodyfixed sensors: outdoor measurement and clinical applications. Comput Animat Virtual Worlds 15(2):79-94

Axisa F, Schmitt PM, Gehin C, Delhomme G, McAdams E, Dittmar A (2005) Flexible technologies and smart clothing for citizen medicine, home healthcare, and disease prevention. IEEE Trans Inf Technol Biomed 9(3):325-336

Beauchet O, Kressig RW, Najafi B, Aminian K, Dubost V, Mourey F (2003) Age-related decline of gait control under a dualtask condition. J Am Geriatr Soc 51(8):1187-1188

Berg KO, Wood-Dauphinee SL, Williams JI, Maki B (1992) Measuring balance in the elderly: validation of an instrument. Can J Public Health 83(Suppl 2):7-11

Bergland A, Pettersen AM, Laake K (1998) Falls reported among elderly Norwegians living at home. Physiother Res Int 3:3-74

Bouten CV, Westerterp KR, Verduin M, Janssen JD (1994) Assessment of energy expenditure for physical activity using a triaxial accelerometer. Med Sci Sports Exerc 26(12):15161523

Buchser E, Paraschiv-Ionescu A, Durrer A, Depierraz B, Aminian K, Najafi B, Rutschmann B (2005) Improved physical activity in patients treated for chronic pain by spinal cord stimulation. Neuromodulation 8:40-48

Bussmann JBJ, Veltink PH, Koelma F, van Lummel RC, Stam HJ (1995) Ambulatory monitoring of mobility-related activities: the initial phase of the development of an activity monitor. Eur J Phys Med Rehabil 5(1):2-7

Collen FM, Wade DT, Robb GF, Bradshaw CM (1991) The rivermead mobility index: a further development of the rivermead motor assessment. Int Disabil Stud 13(2):50-54

Dejnabadi H, Jolles BM, Aminian K (2005) A new approach to accurate measurement of uniaxial joint angles based on a combination of accelerometers and gyroscopes. IEEE Trans Biomed Eng 52(8):1478-1484

Dejnabadi H, Jolles BM, Casanova E, Fua P, Aminian K (2006) Estimation and visualization of sagittal kinematics of lower limbs orientation using body-fixed sensors. IEEE Trans Biomed Eng 53:1385-1393

Dubost V, Herrmann F, Kressig RW, Aminian K, Najafi B, Beauchet O (2006) Relationships between dual-task-related changes in stride velocity and stride time in healthy older adults. Hum Mov Sci 25:372-382

Favre J, Jolles BM, Siegrist O, Aminian K (2006) Quaternion based fusion of gyroscopes and accelerometers to improve 3D angles measurement. Electron Lett 42:612

Hauer K, Lamb SE, Jørstad EC, Todd C, Becker C (2006) Systematic review of definitions and methods of measuring falls in randomised controlled fall prevention trials. Age Ageing 53:5-10
Hausdorff JM, Ladin Z, Wei JY (1995) Footswitch system for measurement of the temporal parameters of gait. J Biom 28(3):347-351

Hausdorff JM, Edelberg HK, Mitchell SL, Goldberger AL, Wei JY (1997) Increased gait unsteadiness in community-dwelling elderly fallers. Arch Phys Med Rehabil 78:278-283

Hausdorff JM, Ashkenazy Y, Peng CK, Ivanov PC, Stanley HE, Goldberger AL (2001) When human walking becomes random walking: fractal analysis and modeling of gait rhythm fluctuations. Physica A 302(1-4):138-147

de Hoon EW, Allum JH, Carpenter MG, Salis C, Bloem BR, Conzelmann M, Bischoff HA (2003) Quantitative assessment of the stops walking while talking test in the elderly. Arch Phys Med Rehabil 84(6):838-842

Kanten DN, Mulrow CD, Gerety MB, Lichtenstein MJ, Aguilar C, Cornell JE (1993) Falls: an examination of three reporting methods in nursing homes. J Am Geriatr Soc 41(6):662-666

Keijsers NL, Horstink MW, Gielen SC (2006) Ambulatory motor assessment in Parkinson's disease. Mov Disord 21:34-44

Lakany HM (2000) A generic kinematic pattern for human walking. Neurocomput 35:27-54

Lindemann U, Hock A, Stuber M, Keck W, Becker C (2005) Evaluation of a fall detector based on accelerometers: a pilot study. Med Biol Eng Comput 43:548-551

Lord SR, Murray SM, Chapman K, Munro B, Tiedemann A (2002) Sit-to-stand performance depends on sensation, speed, balance, and psychological status in addition to strength in older people. J Gerontol Med Sci 57A:M539M543

Luinge HJ, Veltink PH (2005) Measuring orientation of human body segments using miniature gyroscopes and accelerometers. Med Biol Eng Comput 43(2):273-282

Lundin-Olsson L, Nyberg L, Gustafson Y (1997) "Stops walking when talking" as a predictor of falls in elderly people. Lancet 349(9059):1180

Marculescu D, Marculescu R, Park S, Jayaraman S (2003) Ready to ware. IEEE Spec 40(10):28-32

Moe-Nilssen R, Helbostad JL (2005) Interstride trunk acceleration variability but not step width variability can differentiate between fit and frail older adults. Gait Posture 21(2):164-170

Morlock M, Schneider E, Bluhm A, Vollmer M, Bergmann G, Müller V, Honl M (2001) Duration and frequency of every day activities in total hip patients, J Biomech 34:873-881

Mulder T, Zijlstra W, Geurts ACH (2002) Assessment of motor recovery and decline. Gait Posture 16:198-210

Najafi B, Aminian K, Loew F, Blanc Y, Robert P. (2002) Measurement of stand-sit and sit-stand transitions using a miniature gyroscope and its application in fall risk evaluation in the elderly. IEEE Trans Biomed Eng 49(8):843-851

Najafi B, Aminian K, Paraschiv-Ionescu A, Loew F, Bula C, Robert $\mathrm{Ph}$ (2003a) Ambulatory system for human motion analysis using a kinematic sensor: monitoring of daily physical activity in elderly. IEEE Trans Biomed Eng 50(6):711-723

Najafi B, Büla C, Piot-Ziegler C, Demierre M, Aminian K (2003b) Relationship between fear of falling and spatio-temporal parameters of gait in elderly persons, MCC2003 Book, from basic motor control to functional recovery III, chapter II: from posture to Gait pp 152-158

O'Loughlin J, Robitaille Y, Boivin J, Suissa S (1993) Incidence of and risk factors for falls and injurious falls among the community-dwelling elderly. Am J Epidemiol 137:342-354

Pai YC, Rogers MW (1990) Control of body mass transfer as a function of speed of ascent in sit to stand. Med Sc Sports Exerc 22:378-384

Paraschiv-Ionescu A, Buchser EE, Rutschmann B, Najafi B, Aminian K (2004) Ambulatory system for the quantitative 
and qualitative analysis of gait and posture in chronic pain patients treated with spinal cord stimulation. Gait Posture 20(2):113-125

Ploutz-Snyder LL, Manini T, Ploutz-Snyder RJ, Wolf DA (2002) Functionally relevant thresholds of quadriceps femoris strength. J Gerontol Med Sci 57A:M144-M152

Podsiadlo D, Richardson S (1991) The timed "Up \& Go": a test of basic functional mobility for frail elderly persons. J Am Geriatr Soc 39(2):142-148

Roetenberg D, Luinge HJ, Baten CT, Veltink PH (2005) Compensation of magnetic disturbances improves inertial and magnetic sensing of human body segment orientation. IEEE Trans Neural Syst Rehabil Eng 13(3):395-405

Sallis JF, Haskell WL, Wood PD, Fortmann SP, Rogers T, Blair SN, Paffenbarger RS (1985) Physical activity assessment methodology in the Five-City Project. Am J Epidemiol 121(1):91-106

Schenkman M, Hughes M, Samsa G, Studenski S (1996) The relative importance of strength and balance in chair rise by functionally impaired older individuals. J Am Geriatr Soc 44(12):1441-1446

Sekine M, Tamura T, Togawa T, Fuku Y (2000) Classification of waist-acceleration signals in a continuous walking record. Med Eng Phys 22:285-291

Skelton DA, Todd C (2004) What are the main risk factors for falls amongst older people and what are the most effective interventions to prevent these falls? How should interventions to prevent falls be implemented? Health Evidence Network, World Health Organisation, Denmark

Skelton DA, Becker C, Lamb SE, Close JCT, Zijlstra, Yardley L, Todd CJ (2004) Prevention of falls network Europe: a thematic network aimed at introducing good practice in effective falls prevention across Europe. Eur J Ageing 1:89-94
Swartz AM, Strath SJ, Bassett DR, O'Brien WL, King GA, Ainsworth BE (2000) Estimation of energy expenditure using CSA accelerometers at hip and wrist sites. Med Sci Sports Exerc 32(9 Suppl):S450-S456

Terrier P, Aminian K, Schutz Y (2001) Can accelerometry accurately predict the energy cost of uphill/downhill walking? Ergonomics 44(1):48-62

Veltink PH., Bussmann HBJ, de Vries W, Martens WLJ, van Lummel RC (1996) Detection of static and dynamic activities using uniaxial accelerometers. IEEE Trans Rehabil Eng 4(4):375-385

Ware JE Jr, Sherbourne CD (1992) The MOS 36-item short-form health survey (SF-36). I. Conceptual framework and item selection. Med Care 30(6):473-483

Washburn RA, Smih KW, Jette AM, Janey CA (1993) The physical activity scale for the elderly (PASE): development and evaluation. J Clin Epidemiol 46:153-162

Wendel-Vos GC, Schuit AJ, Saris WH, Kromhout D (2003) Reproducibility and relative validity of the short questionnaire to assess health-enhancing physical activity. J Clin Epidemiol 56(12):1163-1169

Woolacott M, Shumway-Cooke A (2002) Attention and the control of posture and gait: a review of an emerging area of research. Gait Posture 16(1):1-14

Zijlstra W, Hof AL (2003) Assessment of spatio-temporal gait parameters from trunk accelerations during human walking. Gait Posture 18(2):1-10

Zijlstra W, Bisseling R (2004) Estimation of hip abduction moment based on body fixed sensors. Clin Biomech 19/8:819-827

Zijlstra W (2004) Assessment of spatio-temporal parameters during unconstrained walking. Eur J Appl Physiol 92:39-44 